\title{
High-intensity Interval Training for Older Adults: Safety Issues
}

\author{
Shigenori Ito
}

\section{ABSTRACT}

High-intensity interval training (HIIT) has been reported to become an alternative of moderate-intensity continuous training and adapted even in older adults. However, to implement the use of HIIT for older adults, who are very often associated with life-style related and chronic diseases, safety issues should be considered in the first place. However, at present, the evaluation of the safety of HIIT among older adults is compromised by the limited availability of relevant data due to the low proportion of studies reporting adverse events. In this review, update data regarding safety and check-up methods for older adults are described in submaximal HIIT and spring interval training.

Keywords: Aerobic, check-up, high-intensity interval training, older, sprint interval training, safety.

Submitted : January 29, 2022

Published : February 23, 2022

ISSN: 2593-8339

DOI: $10.24018 /$ ejmed.2022.4.1.1224

S. Ito*

Division of Cardiology, Sankuro Hospital, Toyota, Japan.

(e-mail: shigeito918@gmail.com)

*Corresponding Author

\section{INTRODUCTION}

Recently, the importance of vigorous exercise to improve maximal oxygen consumption $\left(\mathrm{VO}_{2 \max }\right)$ and resultant decrease of mortality and morbidity have been emphasized; the key of exercise protocol is the intensity [1]. Compared with participants with complete lacking of high-intensity training, those performing $>50 \%$ to $75 \%$ of vigorous physical activity to total physical activity showed a $17 \%$ lower allcause mortality, independent of total moderate to vigorous physical activity [1]. If at least a small amount of highintensity training lacks, the progressive loss of muscle mass and associated function with aging and weight gain will worsen the deleterious course of aging [2], [3]. High-intensity interval training (HIIT) has been reported to become an alternative of moderate-intensity continuous training (MCT) [4] and adapted even in older adults [5]-[8]. However, to optimize the use of HIIT for older adults, who are very often associated with life-style related and chronic diseases, safety issues should be considered in the first place.

\section{SAFETY}

Healthy older adults without overt comorbidities, such as cardiovascular disease or orthopedic injuries, could be adapted to submaximal HIIT program [5]. However, even submaximal HIIT training may increase catecholamine secretion during exercise at an intensity over anaerobic threshold [9]; therefore cardiovascular risks could increase and meticulous check-up would be necessary in older adults compared with younger target groups. Adaptation of all-out sprint interval training (SIT) for older adults has been reported by only a few studies with a limited number of subjects [6], [10], [11].
There is an understandable concern regarding the safety of HIIT in older adults with known or occult coronary artery disease (CAD) and/or chronic heart failure (CHF). At present, the evaluation of the safety of HIIT among adults with varied health and disease characteristics is compromised by the limited availability of relevant data due to the low proportion of studies reporting adverse events [12]. Limited safety data of HIIT in CR under supervision is available, which can be referred to older adults because of high frequency of older participants in CR. HIIT/MCT can be performed at a relatively low risk [13] in stable and selected patients. Among 4846 patients with CAD [13], one fatal cardiac arrest during MCT (129456 exercise hours) and two non-fatal cardiac arrests during HIIT (46364 exercise hours) have been reported with no myocardial infarctions. These low event rates preclude a definitive quantitative determination of the risk associated with HIIT [14]. More recently, only one major non-fatal cardiovascular event occurred among 547 participants with 17083 completed HIIT sessions in patients with $\mathrm{CAD}$ or $\mathrm{CHF}$ [15]. No deaths or cardiac events requiring hospitalization was found among 465 HIIT patients and 488 MCT patients within a medically supervised CR setting [16]. Meanwhile, the incidence of acute adverse responses during or within 24 hours post-exercise in a single session of HIIT was approximately $8 \%$ in 156 patients with cardiometabolic disease. Therefore, caution must be taken when prescribing HIIT to patients with cardiometabolic disease [16]. Additional long-term studies assessing the safety of HIIT are mandatory before wide adoption in older individuals with known or suspected CAD, particularly in unsupervised, nonmedical settings [14], [17].

Data on the safety and risk of injury for SIT are limited [12]. Supra maximal sprints are associated with blood pressure elevation, as well as an increase in blood flow, which could pose a risk of dislodging unstable plaques, potentially 
leading to acute myocardial infarction [18], [19]. Redistribution of blood flow (increased flow in muscle followed by decreased flow in visceral organs) may also pose a risk to patients with cardiovascular disease and chronic kidney disease. However, currently SIT has been adopted in healthy young people, irrespective of their athletic or sedentary lifestyle. For these subjects, the cardiovascular risk could be low due to the low incidence of hypertension and/or atherosclerotic disease. For older individuals with lifestylerelated and/or cardiovascular diseases, the potential risk of the SIT protocol has not been evaluated; thus, it should not be adopted for individuals with clinical issues without clearance [20].

\section{CHECK-UP METHODS}

In my opinion, it is not simple to define a clear and/or unique diagnostic check-up for older adults who want to practice HIIT/ SIT/competitive sports, including all-out exercise; there is currently a relative lack of evidence in the literature on this subject. A guideline by [21] could help judge the adoption of HIIT among participants with clinical problems. This guideline summarizes the clinical considerations for HIIT based on the American College of Sports Medicine and American Heart Association statements, including an initial assessment [3], absolute contraindications to participants [22], [23], monitoring checklist, and indications for avoiding/ceasing HIIT [23]. Medical clearance should be sought for all patients with clinical conditions from medical specialists or general practitioners prior to commencing HIIT [21].

In the check-up to participate in HIIT/SIT programs, the guidelines for sports participation in older adults can be applicable because sports include high-intensity exercise during the entire game or at least transiently depending on the type of sport [24]. Although regular exercise might be useful to lower cardiovascular risk factors, even in those aged $>65$ years, cardiovascular risks increase with age. Therefore, precompetition screening is crucial in older populations as well as those with higher cardiovascular risks [25], [26]. The latest recommendations of the European Society of Cardiology (ESC) highlight the importance of defining the cardiovascular profile by means of pre-participation screenings. Indeed, the main pivotal aim of recent guidelines is to minimize the risk of major cardiovascular adverse events, including sudden cardiac arrest/death during physical activity [24]. It is well known that sudden cardiac arrest /death are very often related to myocardial ischemia, which causes acute coronary syndromes, and most likely affect older people. Therefore, discovering subclinical cardiovascular disease, especially $\mathrm{CAD}$, in subjects who are preparing to participate in HIIT training/sports is fundamental [27]. Coronary computed tomography detects occult CAD in almost $20 \%$ of asymptomatic sportsmen aged $\geqq 45$ years after a normal sports medical evaluation that included resting and bicycle exercise electrocardiogram (ECG) [27]. The coronary artery calcium score reveals most of the relevant $\mathrm{CAD}$ with limited additional value of contrast-enhanced coronary computed tomography angiography [27]. Meanwhile, very few studies have assessed the prevalence of CAD in older athletes with a low atherosclerotic risk profile. Other silent enemies for the coronary systems of athletes/HIIT participants may be congenital heart defects, such as the anomalous origin of the coronary arteries and myocardial bridges (MB). The anomalous origin of coronary arteries has a very low prevalence (about $0.44 \%$ ) in adolescents and very rarely causes sudden cardiac death in the population over 40 years old [28]. Instead, the prevalence of MB is higher; however, in adult/senior individuals, it has been shown that the arterial compression in $\mathrm{MB}$ may be directly related to the atherosclerotic burden proximal to the MB. Therefore, the main objective is to discover unknown/silent CAD, as in the MB-free population [29].

The ESC 2020 guidelines on sports and cardiovascular disease have proposed a relatively simplified algorithm for cardiovascular pre-competition assessment in asymptomatic individuals aged $>35$ years [30]. This tool divides the population into low and high cardiovascular disease risk, based on the number of cardiovascular risk factors or sedentary lifestyle. Based on this algorithm, in older athletes with symptoms, pre-participation cardiovascular examinations should include an exercise ECG test. Functional tests or coronary computed tomography angiography should be considered if the exercise stress test is equivocal or if the ECG is uninterpretable. Stress ECG testing can also cover the diagnosis of exercise-induced arrhythmias, evaluation of blood pressure trends during effort, and quantification of preclinical symptoms such as atypical light tightness and/or initial dyspnea [1]. Finally, in the latest ESC 2020 document, the importance of the exercise testing or cardiopulmonary exercise testing was emphasized to assess cardiovascular risk and measure potential performance for elderly "athletes," especially if inexperienced in moderate to vigorous physical activity [31].

\section{CONCLUSION}

HIIT for older adults, irrespective of the exercise mode (submaximal HIIT or SIT), has been investigated for feasibility and efficacy. However, safety issues cannot be overstressed. Because data is limited, future studies should investigate the safety issues more.

\section{CONFLICT OF INTEREST}

Authors declare that they do not have any conflict of interest.

\section{REFERENCES}

[1] Wang Y, Nie J, Ferrari G, Rey-Lopez JP, Rezende LFM. Association of Physical Activity Intensity With Mortality: A National Cohort Study of 403681 US Adults. JAMA Intern Med. 2020.

[2] Metter EJ, Talbot LA, Schrager M, Conwit R. Skeletal muscle strength as a predictor of all-cause mortality in healthy men. J Gerontol A Biol Sci Med Sci. 2002; 57: B359-65.

[3] Riebe D, Franklin BA, Thompson PD, Garber CE, Whitfield GP, Magal M, et al. Updating ACSM's Recommendations for Exercise Preparticipation Health Screening. Med Sci Sports Exerc. 2015; 47: 2473-9.

[4] Ito S. Rationale and optimising of outcomes in high-intensity interval training for health and disease. J Phys Fitness Sports Med. 2021; 10: 151-64.

[5] Núñez Vergara C, Smith Plaza R, Pérez Ramírez N. [Effectiveness of high intensity interval training in the cardiorespiratory capacity of 
people older than 65 years old: A systematic review]. Rev Esp Geriatr Gerontol. 2021.

[6] Adamson SB, Lorimer R, Cobley JN, Babraj JA. Extremely shortduration high-intensity training substantially improves the physical function and self-reported health status of elderly adults. J Am Geriatr Soc. 2014; 62: 1380-1.

[7] Losa-Reyna J, Baltasar-Fernandez I, Alcazar J, Navarro-Cruz R, Garcia-Garcia FJ, Alegre LM, et al. Effect of a short multicomponent exercise intervention focused on muscle power in frail and pre frail elderly: A pilot trial. Exp Gerontol. 2019; 115: 114-21.

[8] Maire J, Faillenet-Maire AF, Grange C, Dugué B, Tordi N, Parratte B, et al. A specific arm-interval exercise program could improve the health status and walking ability of elderly patients after total hip arthroplasty: a pilot study. J Rehabil Med. 2004; 36: 92-4.

[9] Tanabe K, Osada N, Noda K, Yamamoto M, Omiya K, Itoh H, et al. Changes in hemodynamics and catecholamines during single-level exercise at the anaerobic threshold and $120 \%$ of the anaerobic threshold in normal subjects. $J$ Cardiol. 1994; 24: 61-9.

[10] Adamson S, Kavaliauskas M, Yamagishi T, Phillips S, Lorimer R, Babraj J. Extremely short duration sprint interval training improves vascular health in older adults. Sport Sci Health. 2018.

[11] Hedlund M, Lindelof N, Johansson B, Boraxbekk CJ, Rosendahl E. Development and Feasibility of a Regulated, Supramaximal HighIntensity Training Program Adapted for Older Individuals. Front Physiol. 2019; 10: 590.

[12] Campbell WW, Kraus WE, Powell KE, Haskell WL, Janz KF, Jakicic JM, et al. High-Intensity Interval Training for Cardiometabolic Disease Prevention. Med Sci Sports Exerc. 2019; 51: 1220-6.

[13] Rognmo O, Moholdt T, Bakken H, Hole T, Molstad P, Myhr NE, et al. Cardiovascular risk of high- versus moderate-intensity aerobic exercise in coronary heart disease patients. Circulation. 2012; 126: 1436-40.

[14] Thompson PD, Baggish AL, Franklin B, Jaworski C, Riebe D. American college of sports medicine expert consensus statement to update recommendations for screening, staffing, and emergency policies to prevent cardiovascular events at health fitness affilities. Current Sports Medicine Reports. 2020; 19: 223-31.

[15] Wewege MA, Ahn D, Yu J, Liou K, Keech A. High-Intensity Interval Training for Patients With Cardiovascular Disease-Is It Safe? A Systematic Review. J Am Heart Assoc. 2018; 7: e009305.

[16] Hannan AL, Hing W, Simas V, Climstein M, Coombes JS, Jayasinghe $\mathrm{R}$, et al. High-intensity interval training versus moderate-intensity continuous training within cardiac rehabilitation: a systematic review and meta-analysis. Open Access J Sports Med. 2018; 9: 1-17.

[17] Quindry JC, Franklin BA, Chapman M, Humphrey R, Mathis S. Benefits and Risks of High-Intensity Interval Training in Patients With Coronary Artery Disease. Am J Cardiol. 2019; 123: 1370-7.

[18] Franklin BA. Cardiovascular events associated with exercise. The riskprotection paradox. J Cardiopulm Rehabil. 2005; 25: 189-95.

[19] Thompson PD. The cardiovascular complications of vigorous physical activity. Arch Intern Med. 1996; 156: 2297-302.

[20] Ito S. High-intensity interval training for health benefits and care of cardiac diseases - The key to an efficient exercise protocol. World $J$ Cardiol. 2019; 11: 171-88.

[21] Taylor JL, Holland DJ, Spathis JG, Beetham KS, Wisløff U, Keating SE, et al. Guidelines for the delivery and monitoring of high intensity interval training in clinical populations. Prog Cardiovasc Dis. 2019; 62: 140-6.

[22] Fletcher GF, Ades PA, Kligfield P, Arena R, Balady GJ, Bittner VA, et al. Exercise standards for testing and training: a scientific statement from the American Heart Association. Circulation. 2013; 128: 873934.

[23] Medcine ACoS. ACSM guideline for exercie testing and prescription 9th ed. Philadelphia, PA: Lippincott Williams \& Wilkins. 2014.

[24] Gianturco L, Gianturco V. How do you answer a 65-year-old, without a history of cardiac or vascular disease, when they ask, "Can I participate in competitive sports? ESC E-journal of Cardiology Pracice. 2021; 19.

[25] Chugh SS, Weiss JB. Sudden cardiac death in the older athlete. J Am Coll Cardiol. 2015; 65: 493-502.

[26] Thompson PD, Franklin BA, Balady GJ, Blair SN, Corrado D, Estes NA, 3rd, et al. Exercise and acute cardiovascular events placing the risks into perspective: a scientific statement from the American Heart Association Council on Nutrition, Physical Activity, and Metabolism and the Council on Clinical Cardiology. Circulation. 2007; 115: 235868.

[27] Braber TL, Mosterd A, Prakken NH, Rienks R, Nathoe HM, Mali WP, et al. Occult coronary artery disease in middle-aged sportsmen with a low cardiovascular risk score: The Measuring Athlete's Risk of Cardiovascular Events (MARC) study. Eur J Prev Cardiol. 2016; 23 : 1677-84.
[28] Peñalver JM, Mosca RS, Weitz D, Phoon CK. Anomalous aortic origin of coronary arteries from the opposite sinus: a critical appraisal of risk. BMC Cardiovasc Disord. 2012; 12: 83.

[29] Gowd BM, Thompson PD. Isolated myocardial bridging and exerciserelated cardiac events. Int J Sports Med. 2014; 35: 1145-50.

[30] Pelliccia A, Sharma S, Gati S, Bäck M, Börjesson M, Caselli S, et al. 2020 ESC Guidelines on sports cardiology and exercise in patients with cardiovascular disease. Eur Heart J. 2021; 42: 17-96.

[31] Mont L, Pelliccia A, Sharma S, Biffi A, Borjesson M, Brugada Terradellas J, et al. Pre-participation cardiovascular evaluation for athletic participants to prevent sudden death: Position paper from the EHRA and the EACPR, branches of the ESC. Endorsed by APHRS, HRS, and SOLAECE. Eur J Prev Cardiol. 2017; 24: 41-69. 